Revista Chilena
DE Pediatría

www.revistachilenadepediatria.cl

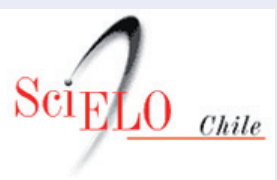

www.scielo.cl

\title{
Guía de práctica clínica Ibero-Latinoamericana sobre la esofagitis cáustica en Pediatría: Aspectos terapéuticos (2ª. Parte)
}

\author{
Ibero-Latinamerican Clinical Practical Guidelines on pediatric caustic esophagitis: \\ Therapeutical aspects (Part 2)
}

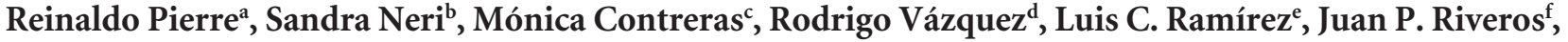 \\ Lisset Rondón ${ }^{\mathrm{g}}$, Adolfo Bautista Casasnovas ${ }^{\mathrm{h}}$, Alfonso Rodríguez-Herrera ${ }^{\mathrm{i}}$, María Navalón', Pilar Soto ${ }^{\mathrm{k}}$, \\ Claudio Iglesias ${ }^{1}$, Giselle Fernández ${ }^{\mathrm{m}}$, Jorge A. Dias ${ }^{\mathrm{n}}$, Carlos J. Ruiz ${ }^{\mathrm{o}}$, José Spolidoro ${ }^{\mathrm{p}}$, Juan Jorge ${ }^{\mathrm{q}}$, Víctor Vila ${ }^{\mathrm{o}}$
}

\author{
aClínica Razetti de Barquisimeto, Barquisimeto, Venezuela \\ bHospital de Niños "Dr. JM de los Ríos", Caracas, Venezuela \\ cHospital de Pediatría S.A.M.I.C. "Prof. Dr. Juan P. Garrahan", Buenos Aires, Argentina \\ 'Hospital Infantil de México Federico Gómez, México DF, México \\ eHospital Federico Lleras Acosta, Ibagué, Colombia \\ ${ }^{\mathrm{f}}$ GastriCare Kids, Bogotá, Colombia \\ Instituto de Ortopedia Infantil Roosevelt, Bogotá, Colombia

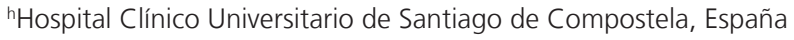 \\ 'Grupo IHP, España \\ 'Hospital Universitario Virgen de la Arrixaca, Murcia, España \\ ${ }^{k}$ Hospital Metropolitano de la Florida, Santiago, Chile \\ 'Hospital Pediátrico, Centro Hospitalario Pereira Rossell, Montevideo, Uruguay \\ mospital Infantil Dr. Robert Reid Cabral, Santo Domingo, República Dominicana \\ ${ }^{n}$ Centro Hospitalario S. Joao, Porto, Portugal \\ - Hospital Sant Joan de Deu, Barcelona, España \\ pNúcleo de Formación em Pediatria da Escola de Medicina da PUCRS, Porto Alegre, Brasil \\ ${ }^{\natural}$ Centro Gastrodiagnóstico y Especialidades, Santo Domingo, República Dominicana
}

Recibido: 11 de junio de 2019; Aceptado: 3 de octubre de 2019

¿Qué se sabe del tema que trata este estudio?

El tratamiento de la esofagitis caustica en el niño todavia constituye un tema dificil con practicas no estandarizadas y recomendaciones muchas veces basadas en evidencia debil. Muchos aspectos del tratamiento de esta patologia todavia generan controversia.
¿Qué aporta este estudio a lo ya conocido?

La presente Guia de Practica Clinica Ibero-Latinoamericana sobre la Esofagitis caustica en Pediatria ofrece una revision sistematica de toda la literatura publicada sobre el tema y una serie de recomendaciones terapeuticas basadas en la evidencia.

Correspondencia:

Reinaldo Pierre

vice.presidencia.laspghan@gmail.com 


\section{Resumen}

La ingestión de cáusticos representa un grave problema médico-social por las consecuencias devastadoras e irreversibles que puede producir en el tracto digestivo superior. En Iberoamérica no se han publicado datos fidedignos sobre la incidencia o la prevalencia de lesiones inducidas por cáusticos. La información disponible sobre la presentación clínica, diagnóstico, tratamiento y pronóstico se basa en series retrospectivas de casos y, de hecho, su manejo clínico se sustenta en muchos casos fundamentalmente en la opinión de expertos. Recientemente como una iniciativa de la Sociedad Latinoamericana de Gastroenterología, Hepatología y Nutrición Pediátrica (SLAGHNP) y con la colaboración de colegas de la Sociedad Española de Gastroenterología, Hepatología y Nutrición Pediátrica (SEGHNP), hemos diseñado una Guía de Práctica Clínica (GPC) la cual incluye una serie de enunciados y recomendaciones dirigidos a optimizar la atención a los pacientes y que se basan en la revisión sistemática de la evidencia. En dos (2) manuscritos sucesivos nos hemos enfocado primero, en los aspectos fisiopatológicos y de diagnóstico clínico-endoscópico de la esofagitis cáustica en niños ( $1^{\mathrm{a}}$. Parte) y en segundo lugar, en los aspectos más relevantes del tratamiento (2a. Parte). Esperamos esta guía se convierta en una herramienta útil para el clínico en el difícil proceso de toma de decisiones a la hora de evaluar un paciente posterior a la ingesta de una sustancia cáustica.
Palabras clave:

esofagitis;

cáustico;

guía de práctica clínica; diagnóstico;

tratamiento

\begin{abstract}
Caustic ingestion represents a serious social-medical problem due to the devastating and irreversible consequences it can produce in the upper digestive tract. In Ibero-America, there are no published reliable data on the incidence or prevalence of caustic-induced injuries, and most of the available information on clinical presentation, diagnosis, treatment, and prognosis is based on retrospective clinical series and, indeed, its clinical management is often based primarily on expert opinion. Recently as an initiative of the Latin American Society for Pediatric Gastroenterology, Hepatology and Nutrition (LASPGHAN) and with the cooperation of the Spanish Society for Pediatric Gastroenterology, Hepatology and Nutrition (SEGHNP), we have designed a Clinical Practice Guideline that include a series of statements and recommendations aimed at optimizing patient medical care which is based on the systematic review of evidence. Two (2) separate papers focused on the evaluation of physiopathological and clinical-endoscopic diagnostic features of caustic esophagitis in children ( $1^{\text {st }}$. Paper) and, on the other hand, the most relevant therapeutic considerations ( $2^{\text {nd }}$. Paper). We expect this guideline to become a useful tool for the physician in the difficult decision-making process when assessing patients after caustic ingestion.
\end{abstract}

\section{Keywords:} esophagitis; caustic; clinical practice guideline; diagnosis; treatment

\section{Introducción}

La ingestión de cáusticos en niños constituye un problema de salud pública, especialmente en los países en vías de desarrollo, y un importante problema médico-social debido a las consecuencias devastadoras e irreversibles sobre la integridad física del niño. En Iberoamérica no se han publicado datos fidedignos sobre la incidencia, prevalencia o mortalidad de las lesiones inducidas por cáusticos. La información disponible sobre la presentación clínica, diagnóstico, tratamiento y pronóstico se basa generalmente en series retrospectivas de casos y estudios no controlados. Es por esta razón que como una iniciativa de las Sociedades Latinoamericana (SLAGHNP) y Española (SEGHNP) de Gastroenterología, Hepatología y Nutrición Pediátrica se formó en el año 2018 un grupo de trabajo con el objetivo de elaborar una guía de práctica clínica (GPC), la cual contiene una serie de enunciados relacionados con fisiopatología, clínica, diagnóstico y tratamiento de la esofagitis cáustica en Pediatría (pacientes menores de 18 años) y para cada uno de los cuales se ha desarrollado un argumento explicativo que conlleva a una serie de recomendaciones. El objetivo del presente trabajo es establecer recomendaciones que puedan orientar al clínico en el difícil proceso de toma de decisiones a la hora de evaluar un paciente posterior a la ingesta de una sustancia cáustica.

\section{Métodos}

Tomando como modelo el concepto de Medicina Basada en Evidencia (MBE) hemos decidido a través del presente manuscrito realizar un análisis de la evidencia disponible desde la perspectiva de los diferentes escenarios clínicos y establecer grados de recomendación para el ejercicio de procedimientos diagnósticos. 
En nuestro caso particular y una vez establecidos los enunciados que formarían parte de la guía, decidimos trabajar con el sistema Grading of Recommendations, Assessment, Development and Evaluation (GRADE) (1), el cual se caracteriza por valorar la evidencia según el área temática o escenario clínico y el tipo de estudio que involucra al problema clínico en cuestión. En el sistema GRADE la calidad de la evidencia se clasifica, inicialmente, en alta ó baja, según provenga de estudios experimentales u observacionales, posteriormente, según una serie de consideraciones, el nivel de evidencia queda en alto, moderado, bajo ó muy bajo.

La fuerza de las recomendaciones puede ser fuerte ó débil y se apoya no sólo en la calidad de la evidencia, sino en una serie de factores tales como el balance entre riesgo y beneficio, la opinión de los expertos y el consumo de recursos o costos ${ }^{1}$.

Se revisaron las principales publicaciones científicas clasificándolas de acuerdo a diseño experimental (estudios experimentales, descriptivos, de cohorte, revisiones sistemáticas, meta-análisis) y de acuerdo a subtemas específicos (fisiopatología, clínica, diagnóstico, tratamiento, etc.). Se dio preferencia a artículos publicados en los últimos 5 años en idioma español, portugués o inglés y a aquellos de mayor nivel de evidencia. En el caso de publicaciones de mucha rigurosidad estadística y evidencia de alta calidad se decidió incluir trabajos de mayor antigüedad. Los buscadores utilizados fueron Bireme, MedLine, Pubmed, Lilacs, Scielo y la Red Cochrane.

La búsqueda se hizo mediante el procedimiento de palabras clave (esofagitis, cáustica, diseño experimental, niños).

En dos (2) manuscritos sucesivos hemos evaluado en primer lugar, aspectos de la fisiopatología y el diagnóstico clínico-endoscópico de la esofagitis cáustica en niños, y en segundo lugar, todo lo relacionado con el tratamiento. Los presentes manuscritos fueron evaluados y aprobados por los miembros del Grupo de Trabajo y por un grupo de especialistas en el área que actuaron como revisores externos.

\section{Tratamiento}

Zargar y col. en el año 1991 publicaron una modificación a la clasificación donde los grados de quemadura cáustica 2 y 3 los subdividieron en $2 \mathrm{~A}, 2 \mathrm{~B}$ y $3 \mathrm{~A}, 3 \mathrm{~B}$, tomando en cuenta la profundidad o lo circunferencial de las úlceras en la 2 y la extensión de la necrosis en la 3. En su publicación demostraron que las lesiones $2 B$ y 3 son predictoras de estenosis ${ }^{2}$. El riesgo de estenosis en esofagitis grado $2 \mathrm{~B}$ es de $32 \%$ de acuerdo a los resultados de una publicación reciente y puede ser hasta de $75 \%$ en niños con esofagitis grado $3^{3}$.
El uso de antibióticos para la prevención de complicaciones infecciosas es controversial. Sólo un trabajo de Krey y col. mostró buenos resultados. Estarían indicados en lesiones grado 3 por riesgo de microabscesos y en los casos de perforación ${ }^{4}$ (Recomendación débil, nivel de evidencia bajo). La inhibición de la secreción ácida mediante el uso de inhibidores de la bomba de protones sí parece estar indicada de forma universal de manera de evitar que el daño péptico secundario al trastorno motor y al reflujo gastroesofágico postquemadura se sume a la lesión cáustica. Sin embargo, no se han realizado ensayos controlados en niños que hayan confirmado la eficacia de este tratamiento. Además, ningún estudio ha determinado la dosis óptima o la duración del tratamiento. En cuanto al uso de corticosteroides en estos casos graves con el objetivo de disminuir las lesiones esofágicas y la incidencia de estenosis, así como la realización de dilataciones de forma precoz son temas controversiales y serán discutidos en párrafos subsiguientes.

Una vez establecido el grado de severidad por endoscopia, el tratamiento debe estar orientado a evitar complicaciones inmediatas, mediatas y tardías. Recomendación Fuerte, nivel de evidencia moderado

Este es un principio universalmente aceptado, del que no encontramos estudios comparativos. Se acepta en toda la literatura revisada, que los pacientes con lesiones leves y hasta aquellos con lesiones grado $2 \mathrm{~A}$ deben permanecer hospitalizados hasta que toleran la alimentación oral completa ${ }^{5-10}$. Sobre los pacientes con lesiones grado $2 \mathrm{~B}$ y 3 , se afirma igualmente que requieren evaluaciones adicionales y el manejo del posible desarrollo de complicaciones mediatas y tardías, una vez superada la fase aguda. La hospitalización inicial habitual es de 2-4 semanas, hasta que la ingesta oral sea óptima ${ }^{10-12}$.

El uso de dexametasona endovenosa ha demostrado ser beneficioso en esofagitis cáustica grado 2B. Recomendación Fuerte, nivel de evidencia moderado

Fulton y Hoffman ${ }^{13}$, analizaron distintos estudios con 328 pacientes con quemaduras esofágicas grado 2 , incluyendo 244 que recibieron terapia con distintos esteroides (dexametasona, Depomedrol ${ }^{\circledR}$, prednisona, prednisolona, metilprednisolona, cortisona e hidrocortisona). Hubo tres estudios prospectivos ${ }^{14-17}$ y ocho retrospectivos. El porcentaje global de estenosis en los grupos tratados y no tratados con esteroides fue del $12,3 \%$ y $19,0 \%$, respectivamente, sin significancia estadística. Solo los estudios de Bautista ${ }^{16}$ y Cadranel $^{18}$ son estudios comparativos entre la utilización de dexametasona y prednisolona, pero no comparan pacientes 
tratados con dexametasona y pacientes no tratados. Las sociedades ESPGHAN y ESGE ${ }^{8,16}$, así como distintos tratados y guías de prestigio, recomiendan su utilización. Se recomienda la administración de altas dosis de dexametasona intravenosa $\left(1 \mathrm{~g} / 1,73 \mathrm{~m}^{2} /\right.$ día $)$ durante 3 días, exclusivamente en la esofagitis cáustica $2 \mathrm{~B}$ como método para prevenir el desarrollo de estenosis esofágica. $1 \mathrm{mg}$ de dexametasona equivale a $6,25 \mathrm{mg}$ de prednisolona, por lo que algún estudio reciente con prednisolona a altas dosis como el de Usta et al. ${ }^{19}$, en el que se demuestra el beneficio de los esteroides a altas dosis, pudiera asimilarse en este punto.

Ni los estudios incluidos en nuestro análisis ni dos recientes revisiones ${ }^{9,20}$ informan de forma rutinaria sobre la aparición de riesgos o efectos adversos inherentes a la terapia con esteroides en los pacientes con esofagitis cáustica 2B.

Preservar el estado nutricional del paciente es otro objetivo del tratamiento y para ello existen diversas alternativas a considerar de acuerdo a la severidad y características del caso. Recomendación débil, nivel de evidencia bajo

Un manejo nutricional individualizado es fundamental. La nutrición es uno de los aspectos terapéuticos más importante ya que contribuye en gran medida a la recuperación más rápida de las lesiones del tracto digestivo, la estabilización de los parámetros biológicos, inmunológicos y metabólicos, además de lograr disminuir la estancia en hospitalización. El soporte nutricional temprano es de gran importancia porque reduce las infecciones y el riesgo de desarrollar neumonía por aspiración y embolismo pulmonar ${ }^{21,22}$.

El tipo de apoyo nutricional va a depender del grado de lesión esofágica que se visualice en la endoscopia (ver clasificación de Zargar):

1) Grado 0: El paciente es dado de alta en forma inmediata $^{23,24}$.

2) Grado 1-2A: Se recomienda la observación intrahospitalaria, no requiere tratamiento específico, los pacientes pueden consumir líquidos y progresar a la comida regular a las $24-48$ horas $^{23,25}$, siendo ésta la conducta más frecuente en la mayoría de los centros. Algunos pocos estudios como el de María Losada y cols. consideran el uso de la nutrición parenteral total durante las primeras 48 horas en este grupo de pacientes ${ }^{21}$.

3) Grado 2B-3: El manejo nutricional en estos pacientes es controversial. Montoro y cols. consideran dieta absoluta por 24-48 horas, fluidoterapia con reposición de líquidos y electrolitos. Es posible considerar en estos casos la colocación de una sonda de alimentación al momento de la endoscopia a pesar del inicio de la alimentación oral. Luego, intentar tolerancia oral posterior a las 48 horas con dieta líquida y posteriormente progresar a dieta usual. Si tras las 24-48 horas no es posible asegurar la ingesta por síntomas persistentes de disfagia o la ingesta oral es deficiente se plantea la nutrición parenteral (NP) o la nutrición enteral (NE) por sonda nasogástrica, nasoduodenal (en casos de necrosis gástrica extensa) o gastrostomía ${ }^{25}$.

La NP se asocia a complicaciones relacionadas con el catéter, infecciones, trastornos metabólicos y alteración del equilibrio ácido base. En vista de las complicaciones descritas y los costos de la misma, algunos autores prefieren el acceso enteral para el soporte nutricional ya que se ha asociado con una reducción del 44\% en las complicaciones infecciosas en las unidades de cuidados intensivos. Además la vía enteral es fisiológica, más segura y costo efectiva. La nutrición parenteral debería quedar restringida a los casos que no toleran la $\mathrm{NE}$ o que exhiben un riesgo elevado de perforación, es decir, aquellos en los que la endoscopia revela lesiones grado 3, generalmente relacionadas con la ingestión de una solución cáustica de gran volumen y/o elevada concentración ${ }^{22,26}$.

La sonda de nutrición enteral puede además prevenir adherencias de las heridas tisulares, prevenir las estenosis, incluso actúa como guía para mantener la luz del órgano ${ }^{26}$.

En cuanto a la gastrostomía, el trabajo descriptivo publicado por Contini y cols. incluyó la realización de gastrostomía tanto en los pacientes con esofagitis cáustica grado 2 o 3 como en aquellos en los que no se les hizo endoscopia por miedo a perforación. La gastrostomía pudiera considerarse en aquellos casos cuando el manejo de las soluciones de alimentación a través de las sondas nasogástricas pudiera ser difícil por el estrecho lumen y en aquellos casos cuando la alimentación enteral deba prolongarse por más de 30 días. En estas circunstancias, la gastrostomía es más efectiva y obligatoria para lograr preservar el estado nutricional, con las ventajas de un fácil manejo debido al mayor diámetro del tubo y la posibilidad de un abordaje endoscópico retrógado en caso de estenosis severa ${ }^{24}$.

Se recomiendan las dilataciones precoces en el manejo de los pacientes con esofagitis cáustica grados $2 \mathrm{~B}$ y 3. Estas pueden reducir el riesgo de desarrollo de estenosis y también la severidad de las mismas. Recomendación débil, nivel de evidencia moderado

No hay consenso sobre el tratamiento dilatador en las estenosis cáusticas, la repercusión a futuro y la calidad de vida en éstos pacientes.

Tampoco se ha establecido el momento óptimo para iniciar el tratamiento dilatador siendo esto aún 
motivo de controversias. En los pacientes con esofagitis grado $2 \mathrm{~B}$ y 3 generalmente es recomendado esperar de tres a seis semanas después de la lesión inicial antes de comenzar con el tratamiento dilatador, dado que la realización de una dilatación más temprana aumentaría el riesgo de perforación esofágica.

La guía ESGE-ESPGHAN publicada en 2016 recomienda la dilatación con balón ó bujías en pacientes con estenosis cáusticas cuando éstas aparecen (Recomendación fuerte, nivel de evidencia bajo $)^{8,27}$. No obstante, varios autores han publicado su experiencia sobre el beneficio de la dilatación precoz comenzando las mismas entre los 7 a 28 días después de la ingestión del cáustico $^{28-30}$. Ante lo expuesto revisamos la bibliografía para establecer si la dilatación precoz: a) Previene la formación de estenosis refractarias; b) Reduce el número de procedimientos dilatadores, y c) Está asociada o no a mayor riesgo de complicaciones (perforación esofágica).

Tugrul Tiryaki ${ }^{28}$ en el año 2005 publicó su experiencia en 28 pacientes con injuria cáustica grados $2 \mathrm{~A}$, 2B y 3 y el uso de dilatación con bujías dividiendo a los pacientes en 2 grupos: grupo A (20 pacientes) sometidos a dilatación temprana comenzando en la primera semana y semanalmente durante el primer mes y grupo B (8 pacientes), comenzando las dilataciones luego de la tercera semana y cada dos semanas posteriormente.

En el grupo A las estenosis fueron resueltas después de 6 meses de dilataciones mientras que en el grupo B, 5 pacientes vieron resuelta su estenosis tras 12 meses, $y$ el resto tras 6,18 y 24 meses $(\mathrm{p}<0,05)$. Hubo 4 perforaciones esofágicas: 2 en cada grupo. Los autores concluyen que las dilataciones tempranas no conllevan un mayor grado de perforación y no previenen la formación de estenosis pero reducen el tiempo de resolución.

Posteriormente Uygun ${ }^{29}$ en un estudio retrospectivo recopilando 8 años de experiencia en dilataciones precoces con balón (haciendo hincapié en el control radioscópico durante el procedimiento), publica en 2013 los resultados en 38 niños con estenosis cáustica a quienes se les clasificó en dos grupos: grupo de dilatación temprana según protocolo de la institución (16 pacientes, tiempo de la primera dilatación 7 a 15 días) y el grupo de dilatación tardía (11 pacientes; $>26$ días). La duración del tratamiento hasta lograr el calibre adecuado fue más corto en el grupo de dilatación temprana (17,6 vs 66,7 días), el número de dilataciones y el tiempo de tratamiento en meses fue menor en pacientes con estenosis cortas (4,9 vs 14,3 y 4,5 meses vs 10,6 meses respectivamente) comparados con el grupo de pacientes con estenosis largas. El 1,6\% de los pacientes presentaron complicaciones relacionadas al procedimiento (perforación esofágica) sin establecerse en que grupo ocurrieron.

Un estudio prospectivo ${ }^{31}$ incluyó 32 niños los cua- les fueron divididos en 2 grupos para realización de dilataciones con Savary sin fluoroscopía: grupo A (dilatación precoz: inicio de la dilatación en un tiempo menor a 10 días post ingesta) y grupo B (dilatación tardía: inicio de la dilatación en tiempo mayor a 10 día post ingesta). Se utilizó el índice de dilatación periódica (IDP) para evaluar el beneficio de la dilatación precoz. Se observó desarrollo de estenosis esofágicas en $21 / 32$ (65\%), de los cuales 6/15 (40\%) pertenecían al grupo A y 15/17 (88\%) al grupo B. Se obtuvo un IDP de 3,04 sesiones/meses para el grupo A vs 4,11 sesiones/meses para el grupo B, con una diferencia significativa entre los grupos $(\mathrm{p}=0,0002)$. En el grupo B se presentó estenosis recurrente en $54 \%$ de los casos. Los resultados del estudio muestran que el tiempo empleado para la resolución de la estenosis esofágica fue menor para los pacientes quienes iniciaron dilataciones de forma pre$\mathrm{COZ}$, sin observarse mayor riesgo de perforación en este grupo.

Tras revisar la bibliografía podemos concluir que existe baja calidad de evidencia en la literatura por ser la mayoría de los estudios de carácter retrospectivo, con número pequeño de casos y metodologías diferentes en cuanto a la técnica de dilatación utilizada y el tiempo entre las dilataciones. Hasta el momento no existen trabajos publicados con evidencia científica de alta calidad que permitan recomendar a la dilatación precoz como terapéutica inicial de los pacientes con esofagitis cáustica, aunque existe evidencia a favor de esta práctica. Para establecer su beneficio en pacientes con estenosis cáusticas grados 2 y 3 se necesitaría de futuros estudios controlados aleatorizados y multicéntricos que abarquen un mayor número de casos y donde se establezca desde el inicio un tratamiento estandarizado.

\section{El manejo de las estenosis cáusticas incluye el uso de diferentes tipos de dilatadores y esquemas de dilata- ción, cada uno con indicaciones de acuerdo al tipo de estenosis. Recomendamos el uso individualizado de los diversos dispositivos disponibles de acuerdo a las características de cada caso. Recomendación débil, ni- vel de evidencia bajo}

Basado en estudios observacionales, las dilataciones esofágicas pueden realizarse utilizando dilatadores tipo bujía desplazados longitudinalmente mediante una guía de alambre por vía anterógrada (por ejemplo, dilatadores Savary-Gilliard) o retrógrada (por ejemplo, dilatadores de Tucker). Sin embargo, se ha encontrado también que los dilatadores tipo balón son igualmente efectivos y pueden reducir la cicatrización secundaria mediante una presión radial más suave en lugar de la fuerza de corte longitudinal ${ }^{29}$.

La dilatación con balón tiene varias ventajas según 
algunos autores, pero sin estudios controlados comparando las diferentes técnicas ${ }^{9,24}$. Es segura y eficaz, ya que carece de la fuerza longitudinal de corte asociada con las bujías. Algunos autores consideran que la dilatación con balón guiado por fluoroscopia es más eficaz y segura que la dilatación con balón endoscópico, ésto basado en estudios observacionales no controla$\operatorname{dos}^{9,24,32,33}$. Las tasas de éxito de la dilatación esofágica con balón con guía fluoroscópica en niños y adultos oscila entre 64 y $100 \%{ }^{34}$. Tanto en la dilatación con Savary-Gilliard como en aquellas realizadas con balón se recomienda la progresión lenta de calibre en cada sesión, deteniendo el procedimiento cuando se logra la laceración de la fibrosis estenótica, pasando a un calibre mayor en la siguiente sesión ${ }^{29}$.

Se informa una tasa de perforación de 0,4-17,4\%, mayor que la incidencia en las estenosis congénitas, relacionadas con el reflujo o anastomóticas ${ }^{24,29,32,33}$. Aunque la principal complicación de la dilatación esofágica es la perforación esofágica, el riesgo es relativamente bajo, especialmente durante la dilatación con balón (0-31\%). El intervalo entre las dilataciones generalmente varía entre 1 y 3 semanas, y se espera que de tres a cinco sesiones proporcionen resultados satisfactorios $^{24}$. Se ha propuesto detener las dilataciones luego de cinco a siete sesiones fallidas y considerar la cirugía reconstructiva ${ }^{35}$. Tales decisiones están influenciadas por varios factores relacionados con el paciente (edad, estado nutricional, riesgos operatorios), la experiencia del médico y la disponibilidad de opciones quirúrgicas alternativas.

Se han descrito una serie de factores considerados de mal pronóstico y predictores de pobre respuesta al tratamiento endoscópico con dilataciones. Estos pacientes pueden desarrollar estenosis refractarias las cuales pueden ameritar una terapéutica endoscópica más agresiva ó una resolución quirúrgica. Recomendación débil, nivel de evidencia bajo

En general se considera que las estenosis de presentación tardía, lesión extensa grado 3, ulceración esofágica persistente, una estenosis fibrótica fuerte que craquea con la dilatación, estenosis mayores de $5 \mathrm{cms}$ y una luz esofágica insuficiente a pesar de múltiples dilataciones por espacio de 9-12 meses se consideran factores de mal pronóstico. Se puede considerar como tratamiento fallido con dilataciones cuando no hay mejoría sustancial en el calibre esofágico luego de 3-6 meses de tratamiento 9 . Otras publicaciones consideran como estenosis esofágica refractaria aquella donde no se puede garantizar una ingesta y un estado nutricional óptimo según la edad del niño luego de un máximo de 5 sesiones de dilataciones con un máximo de 4 semanas de intervalo entre ellas y como estenosis recurren- te, aquellas que resultan de una inhabilidad para mantener un diámetro luminal satisfactorio por 4 semanas una vez el diámetro adecuado para una alimentación apropiada ha sido alcanzado.

Se pueden considerar factores de riesgo para el reemplazo esofágico e indicaciones para tratamiento quirúrgico los siguientes ${ }^{36-38}$ :

- Bajo calibre en la primera dilatación.

- Tamaños promedio de dilatación de $24 \mathrm{Fr}$ por 1 mes con poco progreso.

- Calibre máximo alcanzado a los 3 meses de $28 \mathrm{Fr}$.

- Estenosis mayor a $5 \mathrm{cms}$. de longitud.

- Traqueostomía asociada por lesión de hipofaringe, y

- Estenosis múltiples.

El manejo de estenosis cáusticas refractarias incluye la inyección intra-lesional de esteroides y aplicación de Mitomicina C (MMC). Ambas técnicas ofrecen ventajas y constituyen una alternativa en el manejo de casos complejos. Recomendación débil, nivel de evidencia moderado

La MMC es un antibiótico derivado del Streptomices caespitosus, antineoplásico, anti-fibrótico, utilizado para prevenir la formación de cicatriz y la proliferación de fibroblastos en el postoperatorio de especialidades tales como oftalmología, otorrinolaringología y oncología ${ }^{39,40}$.

Al menos dos estudios avalan el uso de Mitomicina $\mathrm{C}$ (MMC) en estenosis esofágicas refractarias con buena calidad de evidencia y recomendación ${ }^{39,41}$. Los protocolos de aplicación utilizan concentraciones que van desde 0,1 a $0,5 \mathrm{mg} / \mathrm{ml}$ y aplicación tópica (2-5 minutos) acompañando los procedimientos de dilatación.

La respuesta al tratamiento evaluada utilizando escalas de disfagia, índices de resolución sintomática, índice periódico de dilataciones, número total de dilataciones y mejoría endoscópica y radiológica, muestran diferencias significativas en relación al placebo y éxito terapéutico en el 60-80 \% de los casos ${ }^{39,40,42-44}$.

En relación a aplicación de esteroide intralesional el procedimiento se realiza con aguja de escleroterapia calibre 22, preparando acetónido de triamcinolona (40 $\mathrm{mg} / \mathrm{ml}$ ) en $1-3 \mathrm{ml}$ de agua destilada e inyectando 0,5-1 ml en los 4 cuadrantes de la zona estenótica durante la dilatación.

En relación a estudios comparativos entre el uso de esteroides versus MMC en población pediátrica, existe al menos un estudio observacional retrospectivo no aleatorizado que apunta hacia una eficacia inferior de la triamcinolona en relación a la aplicación tópica de MMC. La relativa baja eficacia del esteroide intralesional podría explicarse porque su efecto es antiinflamatorio y no anti-cicatricial ${ }^{45,46}$. 


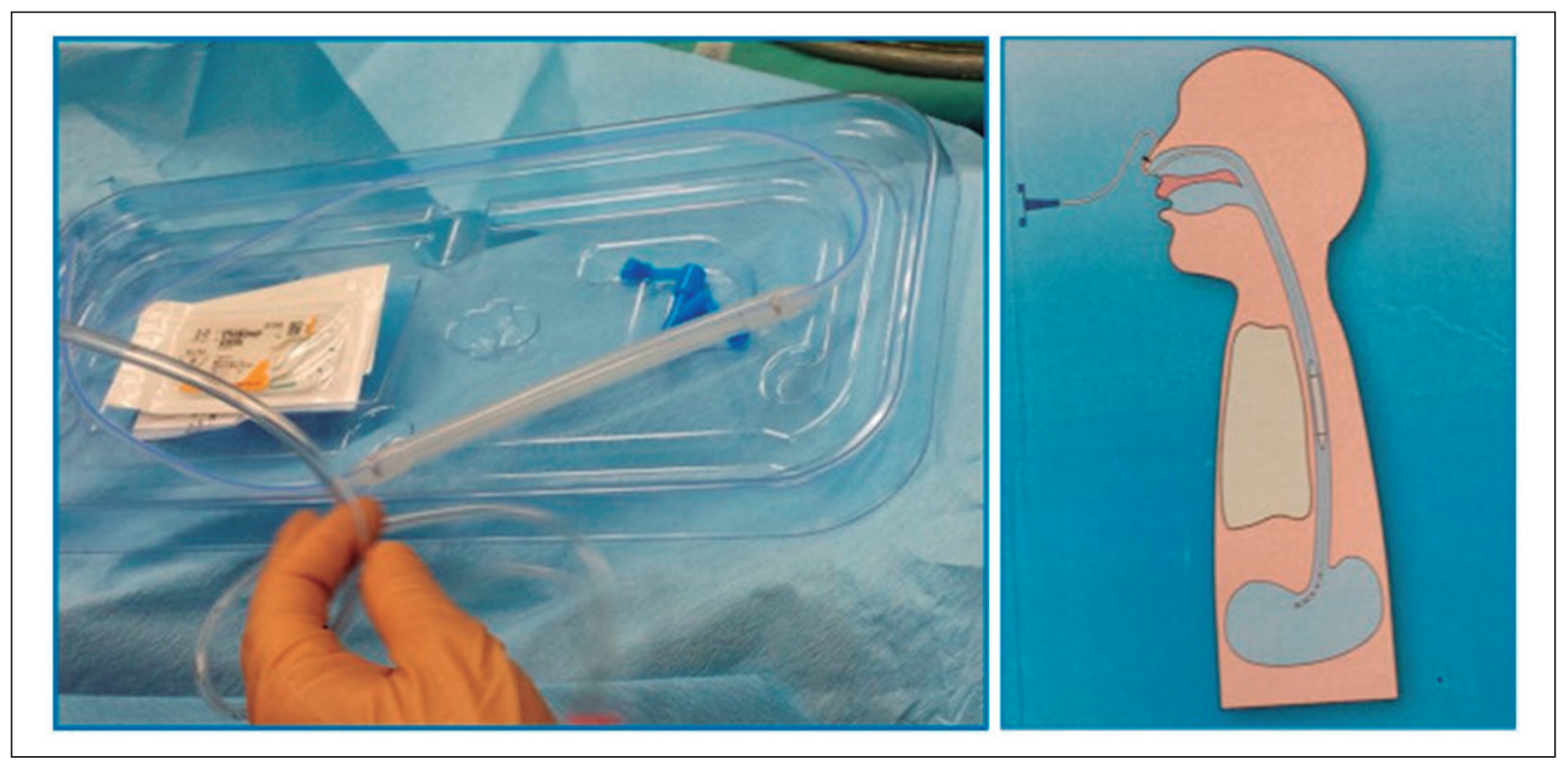

Figura 1. Stent dinámico. Fuente: Abreu M, Nunez I, Corujeira S, Tavares M, Trindade E, Dias JA. Caustic esophageal stenosis: A case report of endoscopic dilation with a dynamic stent. GE Portuguese J Gastroenterol 2016; 23 (4): 218-223.

Los stents esofágicos constituyen una alternativa en pacientes pediátricos con estenosis cáustica refractaria. Su uso es recomendado en pacientes seleccionados. Recomendación débil, nivel de evidencia bajo

En adultos, la colocación de stents es ampliamente conocido, especialmente en el caso de estenosis malig$\mathrm{na}^{47,48}$. A nivel pediátrico, su utilización cada vez es más frecuente, pero existen pocos datos que justifiquen su eficacia y seguridad, especialmente en estenosis secundarias a ingestión de cáustico ${ }^{49}$.

Los más frecuentemente usados son los stents autoexpandibles de metal (SEMS) cubiertos o no, los stents autoexpandibles de plástico (SEPS), los stents biodegradables autoexpandibles y el stent dinámico (figura 1).

En pediatría, los reportes sobre la eficacia del uso de stents para el tratamiento de la estenosis esofágica refractaria benigna están limitados a estudios observacionales, retrospectivos, tipo cohortes y series de casos pequeñas. La tasa global de efectividad en los diferentes estudios pediátricos publicados oscila entre el 46 y el $93 \%$, siendo el stent autoexpandible de metal y el stent dinámico los que han ofrecido mejores resultados ${ }^{50,51}$.

Actualmente, no existe evidencia suficiente que sugiera que el uso de stents sea superior al programa de dilataciones esofágicas. Solo hay un único estudio que de forma retrospectiva ha comparado la eficacia de ambos tratamientos endoscópicos, encontrando una mayor tasa de efectividad con el uso de stents (63\% vs $33 \%$ ) al evaluar la ausencia de síntomas y de recurrencia de la estenosis ${ }^{52}$.
Si bien no hay consenso en relación al número de dilataciones esofágicas que se deben realizar previo al uso del stent, los estudios publicados, con poca evidencia, reportan resultados muy heterogéneos, que oscilan entre 1-45 dilataciones esofágicas realizadas antes de decidir colocar el stent. Un dato muy importante es que los estudios que utilizaron stents de forma temprana (después de 1-3 dilataciones), son los estudios con mayor número de pacientes $(\mathrm{n}=132)$ y mayor tasa de eficacia $(69-93 \%)^{50,52,53}$. Por otro lado, los estudios con uso de stent más tardíamente (después de 4 ó más dilataciones) presentaron tasa de eficacia menor (46$60 \%$ ), siendo la mayoría de ellos, series pequeñas y reportes de $\operatorname{casos}^{54,55}$.

El tiempo adecuado para la colocación del stent después de la ingestión del cáustico no está bien establecido. Hay estudios que consideran que la colocación temprana del stent (antes de los 2 meses), podría prevenir la remodelación del tejido y las estenosis fibróticas resistentes ${ }^{56,57}$. Sin embargo, estos estudios son limitados a reportes de casos, con tamaño muestral insuficiente. Por otro lado, series más grandes reportan una efectividad de 69-72\% con SEMS, cuando se coloca entre 2-22 meses después de la ingestión del cáustico ${ }^{52,53,57}$.

La duración o el tiempo óptimo que debe permanecer colocado el stent en el sitio de la estenosis tampoco ha sido establecido. En adultos suele ser como promedio entre 8-12 semanas y dependerá del tipo y longitud de estenosis, severidad de la inflamación y el tipo de stent utilizado ${ }^{58-60}$. En niños, suele determinarse en general, según el tipo de stent a utilizar, pero 
Tabla 1. Resumen. Enunciados, Recomendaciones y Niveles de evidencia

\begin{tabular}{|c|c|}
\hline \multicolumn{2}{|l|}{ Tratamiento } \\
\hline $\begin{array}{l}\text { Se recomienda tratamiento antibiótico en lesiones Zargar tipo } 3 \text { o superiores por riesgo de microabscesos } \\
\text { y en caso de perforación. }\end{array}$ & $\begin{array}{l}\text { Recomendación débil. } \\
\text { Nivel de evidencia bajo }\end{array}$ \\
\hline $\begin{array}{l}\text { El uso de dexametasona a dosis altas ( } 1 \mathrm{~g} / 1.73 \mathrm{~m} 2 / \mathrm{d} \text { por } 3 \text { días) en lesiones grado } 2 \mathrm{~B} \text { puede disminuir el } \\
\text { riesgo de estenosis esofágica. }\end{array}$ & $\begin{array}{l}\text { Recomendación fuerte. } \\
\text { Nivel de evidencia moderado }\end{array}$ \\
\hline $\begin{array}{l}\text { Se debe mantener un estado nutricional adecuado (por vía oral, soporte enteral, soporte parenteral), según } \\
\text { la severidad de la lesión, para disminuir tiempo de hospitalización y mejorar el pronóstico }\end{array}$ & $\begin{array}{l}\text { Recomendación débil. } \\
\text { Nivel de evidencia bajo }\end{array}$ \\
\hline $\begin{array}{l}\text { Se pueden realizar dilataciones esofágicas precoces (primeras 1-4 semanas) en pacientes con lesiones grado } \\
\text { 2B y } 3 \text { para disminuir el riesgo de estenosis }\end{array}$ & $\begin{array}{l}\text { Recomendación débil. } \\
\text { Nivel de evidencia moderado }\end{array}$ \\
\hline Se recomienda la dilatación con balón o bujías en pacientes con estenosis esofágica. & $\begin{array}{l}\text { Recomendación débil. } \\
\text { Nivel de evidencia bajo }\end{array}$ \\
\hline $\begin{array}{l}\text { Valorar factores predictores de pobre respuesta a tratamiento endoscópico para determinar manejo endos- } \\
\text { cópico adicional o manejo quirúrgico }\end{array}$ & $\begin{array}{l}\text { Recomendación débil. } \\
\text { Nivel de evidencia bajo }\end{array}$ \\
\hline $\begin{array}{l}\text { El manejo de estenosis cáusticas refractarias incluye la inyección intra-lesional de esteroides como la triamci- } \\
\text { nolona y aplicación de Mitomicina C (MMC). Ambas técnicas ofrecen ventajas y constituyen una alternativa } \\
\text { en el manejo de casos complejos. }\end{array}$ & $\begin{array}{l}\text { Recomendación débil. } \\
\text { Nivel de evidencia moderado }\end{array}$ \\
\hline $\begin{array}{l}\text { Los stents esofágicos constituyen una alternativa en pacientes pediátricos con estenosis cáustica refractaria. } \\
\text { Su uso es recomendado en pacientes seleccionados. }\end{array}$ & $\begin{array}{l}\text { Recomendación débil. } \\
\text { Nivel de evidencia bajo }\end{array}$ \\
\hline $\begin{array}{l}\text { Se recomienda vigilancia clínica y endoscópica a largo plazo por riesgo de malignización de esófago en casos } \\
\text { de esofagitis severa o instrumentación recurrente. }\end{array}$ & $\begin{array}{l}\text { Recomendación débil. } \\
\text { Nivel de evidencia bajo }\end{array}$ \\
\hline
\end{tabular}

EDS: Endoscopia digestiva superior; IBP: Inhibidor de la bomba de protones; VO: vía oral; NP: nutrición parenteral; NE: nutrición enteral; ATB: antibióticos; DEE: dilatación esofágica endoscópica.

debido a la pobre calidad de los estudios, no se puede establecer conclusiones adecuadas. En general, no hay suficiente evidencia que sugiera el tiempo adecuado que deben permanecer los stents a nivel de esofágico, así como no hay datos concluyentes sobre la relación entre tiempo de permanencia del stent y su eficacia y seguridad.

Entre las complicaciones relacionadas al uso de stents, la migración es tal vez la complicación más frecuente y significativa. Los estudios reportan una prevalencia $10-30 \%$ aproximadamente $\mathrm{e}^{5,57,61,62}$.

\section{Pronóstico}

Existe un riesgo de malignidad asociado al desarrollo de una esofagitis cáustica severa. Se recomienda en estos casos vigilancia clínica y endoscópica a largo plazo. Recomendación débil, nivel de evidencia bajo

No existen revisiones sistemáticas sobre el riesgo relativo de neoplasia en pacientes con ingesta cáustica. Sólo una publicación que aborda este tema en un escenario epidemiológico y terapéutico completamente diferente al actual podría ser considerada como evidencia ${ }^{63}$. Kay et al ${ }^{64}$ mencionan un aumento del riesgo de neoplasia esofágica (adenocarcinoma y espinocelular) de 2 a $30 \%$, aunque no mencionan la fuente de tales datos. Mamede et al. ${ }^{65}$ mencionan la ocurrencia de 4 casos de neoplasia en 239 pacientes con ingesta cáustica (adultos y niños). Las neoplasias ocurrieron en los adultos a una edad media de 51 años ( 2 casos de ingesta accidental y 2 por intento de suicidio).

Por el contrario, un análisis sistemático sobre el uso de corticoides posterior a ingesta cáustica incluye una revisión de la literatura desde 1956 hasta 2006 y no menciona registro de neoplasias, aunque es necesario aclarar que éste no fue el objetivo primario del estudio $^{13}$. El largo tiempo que puede transcurrir entre el incidente y la aparición de la neoplasia esofágica o gástrica hace difícil establecer una relación de causalidad debido a la posible influencia de otros factores (por ejemplo, tabaco y alcohol) ${ }^{66}$. La descripción de casos clínicos aislados es insuficiente para realizar un análisis sistemático. Diversos trabajos recientes concluyen que el riesgo de neoplasia posterior a una ingesta cáustica ha sido sobrevalorado en serie antiguas o de casos clínicos aislados ${ }^{24,66,67}$.

En principio, la lesión grave de la mucosa esofágica o gástrica y la manipulación instrumental (dilataciones, aplicación tópica de medicamentos, etc.) podría causar inflamación crónica y modificaciones estructurales en tejidos y mucosas. La descripción de casos aislados de neoplasia en pacientes con esofagitis cáustica severa es insuficiente para poder afirmar que en efecto existe un riesgo elevado de desarrollo de cáncer, aunque tampoco es posible asegurar que este riesgo es nulo. 


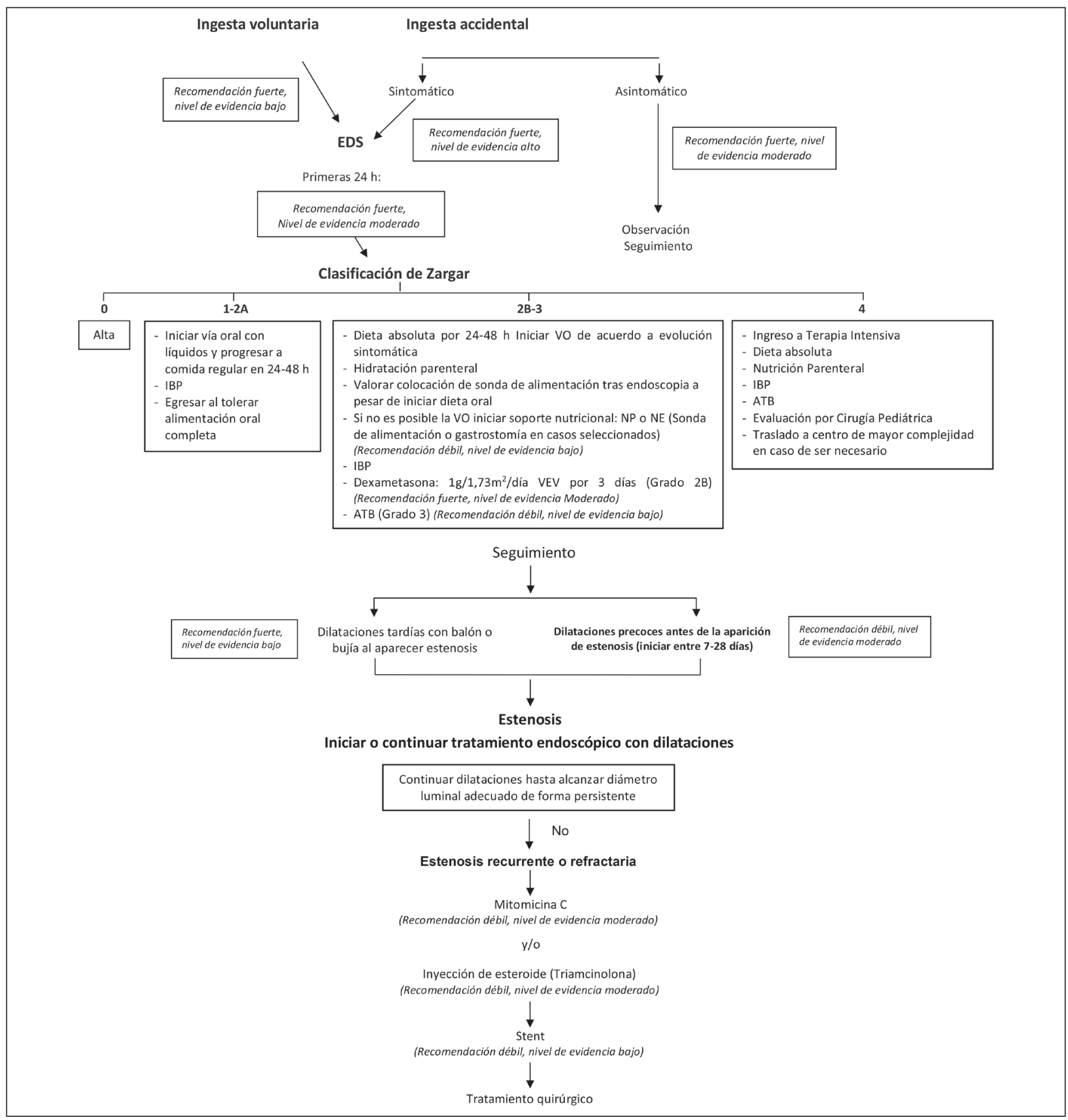

Figura 2. Algoritmo. Diagnóstico y tratamiento Esofagitis cáustica en niños. EDS: Endoscopia digestiva superior; IBP: Inhibidor de la bomba de protones; VO: vía oral; NP: nutrición parenteral; NE: nutrición enteral; ATB: antibióticos; DEE: dilatación esofágica endoscópica.

\section{Conclusiones}

El abordaje terapéutico de la esofagitis cáustica en niños comienza con el diagnóstico endoscópico apropiado e incluye la puesta en práctica de una serie de medidas entre cuyos objetivos están minimizar el daño, preservar el estado nutricional y evitar complicaciones inmediatas, mediatas y tardías. La tabla 1 muestra el resumen de enunciados te- rapéuticos, los niveles de evidencia y grados recomendación respectivos. Asimismo, la revisión de la literatura y el análisis respectivo expuesto en el presente manuscrito nos ha permitido diseñar y proponer un Algoritmo de manejo para todos los casos de ingesta cáustica en niños (figura 2).

\section{Conflicto de intereses}

Los autores declaran no tener conflicto de intereses. 


\section{Referencias}

1. Aguayo-Albasini JL; Flores-Pastor B; Soria-Aledo V. Sistema GRADE: clasificación de la calidad de la evidencia y graduación de la fuerza de la recomendación. Cir Esp. 2014; 92(2):82-8.

2. Zargar SA, Kochhar R, Mehta S, Mehta SK. The role of fiberoptic endoscopy in the management of corrosive ingestion and modified endoscopic classification of burns. Gastrointest Endosc. 1991; 37:165.

3. Cheng HT, Chen CL, Lin CH, et al. Caustic ingestion in adults: the role of endoscopic classification in predicting outcome. BMC Gastroenterology. 2008; 8:31.

4. Rosenberg N, Kunderman PJ, Vroman L, Moolten SE. Prevention of experimental esophageal stricture by cortisone II. Control of suppurative complications by penicillin. AMA Arch Surg. 1953;66(5):593-98.

5. Millar AJ, Cox SG. Caustic injury of the oesophagus. Pediatr Surg Int. 2015;31:111-21.

6. Betalli P, Falchetti D, Giuliani S, et al. Caustic ingestion in children: is endoscopy always indicated? The results of an Italian multicenter observational study. Gastrointest Endosc. 2008;68:434639.

7. Nuutinen M, Ubari M, Kourvalainen $\mathrm{K}$. Consequences of caustic ingestion in children . Acta pediatr. 1994; 83:1200-5.

8. Thomson M, Tringali A, Dumonceau JM, et al. Paediatric Gastrointestinal Endoscopy: European Society for Paediatric Gastroenterology Hepatology and Nutrition and European Society of Gastrointestinal Endoscopy Guidelines. J Pediatr Gastroenterol Nutr. 2017;64(1):133-53.

9. Arnold M, Numanoglu A. Caustic ingestion in children - A review. Seminars in Pediatric Surgery 2017;26:95-104.

10. Maluenda Carrillo C, Bodas Pinedo A. Capitulo 3.1.4 Traumatismos, infecciones y esofagitis cáustica. En Tratado de Gastroenterología, Hepatología y Nutrición Pediátrica aplicada Págs.: 185190, Edit. Ergon, Madrid, 2011.

11. Sánchez-Ramírez CA, Larrosa-Haro A, Vásquez Garibay EM, Larios-Arceo F. Caustic ingestión and oesophageal damage in children: Clinical spectrum and feeding practices. J Ped and Child Health. 2011;47(6):378-80.

12. Ramos Varela JC, González Santana D, Rial González R. Capítulo 3. Ingesta de Cáusticos. En Tratamiento en Gastroenterología, Hepatología y Nutrición Pediátrica. Pags: 45-52, 4a Ed. Editorial Ergon, Madrid. 2016.

13. Fulton JA, Hoffman RS. Steroids in second degree caustic burns of the esophagus: a systematic pooled analysis of fifty years of human data: 1956-2006. Clin Toxicol (Phila). 2007;45(4):402-8.

14. Anderson KD, Rouse TM, Randolph JG. A controlled trial of corticosteroids in children with corrosive injury of the esophagus. N Engl J Med. 1990; 323:637.

15. Hawkins DB, Demeter MJ, Barnett TE. Caustic ingestion: Controversies in management: A review of 214 cases. Laryngoscope. 1980;90:98-108.

16. Bautista A, Varela R, Villanueva A, Estevez E, Tojo R, Cadranel S. Effects of prednisolone and dexamethasone in children with alkali burns of the esophagus. J Pediatr Surg. 1996;6:198-203.

17. Webb WR, Koutras P, Ecker RR, Sugg WL. An evaluation of steroids and antibiotics in caustic burns of the esophagus. Ann Thorac Surg. 1970;9:95101.

18. Cadranel S, Scaillon M, Goyens P, et al. Treatment of esophageal caustic injuries-experience with high-dose dexamethasone. Pediatr Surg Int. 1993;8(2):97-102.

19. Usta M, Erkan T, Cullu Cokugras F, et al. High doses of methylprednisolone in the management of caustic esophageal burns. Pediatrics. 2014;133(6):1518-24.

20. Tringali A, Thomson M, Dumonceau JM, et al. Pediatric gastrointestinal endoscopy: European Society of Gastrointestinal Endoscopy (ESGE) and European Society for Paediatric Gastroenterology Hepatology and Nutrition (ESPGHAN) Guideline Executive summary. Endoscopy. 2017;49(1):83-91.

21. Losada M, Rubio M, Blanca JA, Pérez C. Ingesta de cáusticos en niños, experiencia de 3 años. Rev Chil Pediatr. 2015;86(3):189-93.

22. Chibishev A, Markoski V, Smokovski I, Shikole E, Stevcevska A. Nutricional therapy in the treatment of acute corrosive intoxication in adults. Mater Sociomed. 2016;28(1):66-70.

23. Park KS. Evaluation and Management of Caustic Injuries from Ingestion of Acid or Alkaline Substances. Clin Endosc. 2014;47(4):301-7.

24. Contini S, Scarpignato C. Caustic injury of the upper gastrointestinal tract: a comprehensive review. World J Gastroenterol. 2013;19:3918-30.

25. Montoro, MA. Anexo II. Guía de manejo clínico de las lesiones esofagogástricas por cáusticos. Proyecto REiBECA: Registro Iberoamericano de Lesiones Esofagogástricas Inducidas por Cáusticos. Octubre 2015.

26. Kochhar R, Poornachandra KS, Puri P, et al. Comparative evaluation of nasoenteral feeding and jejunostomy feeding in acute corrosive injury: a retrospective analysis. Gastrointest Endosc. 2009;70(5):874-80.
27. Vandenplas Y. Management of Benign Esophageal Strictures in Children. Pediatr Gastroenterol Hepatol Nutr. 2017;20:2115.

28. Tiryaki T, Livanelioglu Z, Atayurt $\mathrm{H}$ (2005) Early bougienage for relief of stricture formation following caustic esophageal burns. Pediatr Surg Int. 2005; 21(2):78-80.

29. Uygun I, Arslan MS, Aydogdu B, et al. Fluoroscopic balloon dilatation for caustic esophageal stricture in children: an 8-year experience. J Pediatr Surg. 2013; 48:2230.

30. Gun F, Abbasglu L,Celik A, Salman ET. Early and late term management in caustic ingestion in children:a 16 -year experience. Acta Chir Belg. 2007;107(1):49-52.

31. Arrieta A, Navarro D, Durango R, et al. Dilatación precoz: evolución y complicaciones en esofagitis caústicas grado II y III en niños. Gen v.65 n.3 Caracas sep. 2011.

32. Temiz A, Oguzkurt P, Ezer SS, et al. Predictability of outcome of caustic ingestion by esophagogastroduodenoscopy in children. World J Gastroenterol 2012;18:1098-103.

33. Shub MD. Therapy of caustic ingestion: new treatment considerations. Curr Opin Pediatr 2015; 27: 609-613.

34. Uygun I. Caustic esophagitis in children: prevalence, the corrosive agents involved, and management from primary care through to surgery. Current Opinion in Otolayngology and Head and Neck Surgery 2015;23(6):423-32.

35. Chirica M, Resche-Rigon M, Pariente B, et al. Computed tomography evaluation of high-grade esophageal necrosis alter corrosive ingestion to avoid unnecessary esophagectomy. Surg Endosc 2015; 29: 1452-1461.

36. Karaman İ, Koç O, Karaman A, Erdoğan $\mathrm{D}$, et al. Evaluation of 968 children with corrosive substance ingestion. Indian J Crit Care Med. 2015;19(12):714-8.

37. Panieri E, Rode H, Millar AJ, Cywes S. Oesophageal replacement in the management of corrosive strictures: when is surgery indicated?. Pediatr Surg Int. 1998;13(5-6):336-40.

38. El-Asmar K, Hassan, Hesham, Hamza A. Topical mitomycin C application is effective in management of localized caustic esophageal stricture. A doubleblinded randomized, placebo controlled trial. J Ped Surg. 2013;48:1621-7.

39. Türkylmaz Z, Sönmez K, et al. Mitomycin $\mathrm{C}$ prevents strictures in caustic esophageal burns in rats. J Surg Res. 2005;123:182-7.

40. El-Asmar K, Hassan M, Abdelkoder. Topical mitomycin C can effectively alleviate dysphagia in children with long segment caustic esophageal strictures. Dis esophagus; 28. 422-7. 
41. Nagaich N, Nijhawan S. Mitomycin C: A "ray of hope" in refractory corrosive esophageal strictures. Dis esophagus 2014;27:203-205.

42. Rosseneau S, yerushalmi B, ibarguen E, et al. Topical application of mitomycin $\mathrm{C}$ in oseophageal strictures. JPGN 2007;44:336-41.

43. Uhlen S, Fayoux P, Vachin F, et al. Mitomycin C an alternative treatment for refractatory esophageal stricture in children. Endoscopy 2006;38:404-7.

44. Berger M, Ure B, Lacher M. Mitomycin $\mathrm{C}$ is the therapy of recurrent esophageal strictures: hype or hope? Eur J Pediatr Surg. 2012;22:109-16.

45. Nyhawan S, Udawat HP, Nagar P. Aggresive bougie dilatation and intralesional steroids is effective in refractory bening esophageal strictures secondary to corrosive ingestión. Dis esophagus 2016;29(8):1027-31.

46. Méndez C, Ramírez Mayans J, Flores M, et al. Mitomicina C tópica contra triamcinolona intralesional en el manejo de estenosis esofágica por cáusticos. Rev Gastroenterol Mex. 2015;80(4):248-54.

47. Repici A, Hassan C, Sharma P, Conio M, Siersema P. Systematic review: the role of self-expanding plastic stents for benign oesophageal strictures. Aliment Pharmacol Ther. 2010;31:268-1275.

48. Best C, Sudel B, Foker JE, Krosch TC, Dietz C, Khan KM. Esophageal stenting in children: indications, application, effectiveness, and complications. Gastrointest Endosc. 2009;70(6):1248-53

49. Foschia F, De Angelis P, Torroni F, et al. Custom dynamic stent for esophageal strictures in children. J Pediatr Surg. 2011; 46(5):848-53.
50. Mutaf O. Treatment of corrosive esophageal strictures by long-term stenting. J Pediatr Surg. 1996; 31(5):6815.

51. Abreu M, Nunes I, Corujeira S, Tavares M, Trindade E, Dias JA. Caustic Esophageal Stenosis: A Case Report of Endoscopic Dilation with a Dynamic Sten. GE Portuguese J Gastroenterol .2016; 23(4):218-223.

52. Vandenplas Y, Hauser B, Devreker T, Urbain D and Reynaert H. A biodegradable esophageal stent in the treatment of a corrosive esophageal stenosis in a child. J Pediatr Gastroemterol Nutr. 2009;49(2):254-7.

53. Van Boeckel PG and Siersema PD. Refractory esophageal strictures: what to do when dilation fails. Curr Treat Options Gastroenterol. 2015;13(1):47-58.

54. Woynarowski M, Dadalski M, Wojno V, Teisseyre M, Hurkala L, Plowiecki E. Novel, double lumen removable stent to treat caustic esophageal stenosis. Endoscopy 2014;46(1):378-9.

55. Kramer RE, Quiros JA. Esophageal stents for severe strictures in young children: experience, benefits, and risk. Curr Gastroenterol Rep. 2010;12(3):203-10.

56. Coln D, Chang JH. Experience with esophageal stenting for caustic burns in children. J Pediatr Surg 1998;21(7):58891.

57. Atabek C, Surer I, Demirbag S, Caliskan B, Ozturk H, Cetinkursun S. Increasing tendency in caustic esophageal burns an long-term polytetrafluorethylene stenting in severe cases: 10 years experience. J Pediatr Surg 2007;42(4):636-40.

58. Martin Cano F, Rodriguez Vargas J, Velasco Sánchez B, Herrera Montes I. Use of self-expandable prothesis in esophageal stenosis in children. Cir Pediatr. 2012;25(4):207-10.

59. Zheng J, Chang Z, Liu Z, et al. Retrievable Z-stent for the treatment of refractory corrosive esophageal strictures in children. Eur J Pediatr Surg. 2015;25(2):160-4.

60. Broto J, Asensio M and Vernet JM. Results of a new technique in the treatment of severe esophageal stenosis in children: poliflex stents. J Pediatr Gastroenterolol Nutr. 2003;37(2):203-6.

61. Berkovits RN, Bos CE, Wijburg FA and Holzki J. Caustic injury of the oesophagus: Sixteen years experience and introduction of a new model of esophageal stent. J Laryngol Otol. 1996; 110(11):1041-5.

62. Karakan T, Utku OG, Dorukoz O, et al. Biodegradable stents for caustic esophageal strictures: a new therapeutic approach. Dis Esophagus 2013;26(3):31922.

63. Kiviranta NK. Corrosive carcinoma of the esophagus. Acta Otolaryngol 1952; 102: 1-9.

64. Kay M, Willye R. Caustic Ingestion in children. Curr Op Ped 2009, 21:651-4.

65. Mamede RC, de Mello Filho FV. Ingestion of caustic substances and its complications. Sao Paulo Med J 2001;119 (1):10-5.

66. McAuley CE, Steed DL, Webster MW. Late sequelae of gastric acid injury. American journal of surgery. 1985;149(3):412-5.

67. Ntanasis-Stathopoulos I, Triantafyllou S, Xiromeritou V, Bliouras N, Loizou C, Theodorou D. Esophageal remnant cancer 35 years after acidic caustic injury: A case report. Internat J Surg Case Reports 2016;25:21. 